effects on pericyte biology in vitro, yet the significance of this in vivo and in regard to diabetes remains unexplored.

Methods Using a mouse model with a pericyte specific knockout of the insulin receptor (PIR-/-), created by crossing PDGFR $\beta^{2}$-Cre mice with insulin receptor - floxed - mice, we investigated the role of pericyte insulin signalling in developmental retinal angiogenesis in vivo and on pericyte function in vitro.

Results At postnatal day 5, PIR-/- retinas show hyper-vascularized venous regions with an increase in venous vascular density $(57.6 \pm 1.0 \%$ compared to $52.1 \pm 0.6 \%$ in littermate controls, $\mathrm{p}=0.0003$ ) along with an increase in sprouting tip cell formation at the vascular front $(1.7 \pm 0.05$ versus 1.5 \pm 0.06 per $100 \mu \mathrm{m}$ in controls, $\mathrm{p}=0.006)$. Pericyte coverage at postnatal day 5 appears comparable in PIR-/- and control, but pericyte abundance is strikingly reduced by $20 \%$ in the mature adult retinal vasculature $(0.8 \pm 0.03$ and $1.0 \pm 0.05$ per $100 \mu \mathrm{m}$ in controls, $\mathrm{p}=0.002)$. However, the differential vascularity of neonatal retinas did not persist in adulthood $(18.1 \pm 0.7 \mathrm{~mm}$ versus $18.3 \pm 0.5 \mathrm{~mm}$ per $\mathrm{mm}^{2}$ region of interest in control). Basic metabolic phenotyping of adult mice shows no difference in body weight or fasting blood glucose levels $(5.0$ $\pm 0.2 \mathrm{mmol} / \mathrm{L}$ and $4.7 \pm 0.2 \mathrm{mmol} / \mathrm{L}$ in PIR-/- and control mice, respectively), but interestingly, preliminary assessment of pericyte function in vitro suggests enhanced proliferation of PIR-/pericytes.

Conclusions Pericyte-specific insulin receptor knockout affects developmental retinal angiogenesis in vivo. Sprouting and remodelling of the retinal vasculature are altered, associated with a reduction in pericyte coverage in adulthood, recapitulating some characteristics of proliferative diabetic retinopathy. The underlying molecular mechanism remains unclear, but these results indicate that adequate pericyte insulin signalling is important for pericyte function, and hence vascular stability.

\section{NOVEL THIENOPYRIDINES ARE POTENT ANTI-PLATELET DRUGS, INHIBITING PLATELET ACTIVATION, AGGREGATION AND SHOWING SYNERGY WITH ASPIRIN}

${ }^{1}$ Naif Binsaleh*, ${ }^{1}$ Catherine A. Wigley, ${ }^{1}$ Kathryn A. Whitehead, ${ }^{1}$ Daniel Moreno-Martinez, ${ }^{1}$ Sarah Daniels, 'Sarah Jones, ${ }^{2}$ Michelle van Rensburg, ${ }^{2}$ Lisa Pilkington, ${ }^{2}$ David Barker, ${ }^{1}$ Nina Dempsey-Hibbert. 'School of Healthcare Science, Manchester Metropolitan University, Manchester, UK, M1 5GD; ${ }^{2}$ School of Chemical Sciences, The University of Auckland, New Zealand

\subsection{6/heartjnl-2017-311726.185}

Background Management of Acute Coronary Syndromes (ACS) often involves the use of platelet inhibitors. The most commonly used drug, clopidogrel, belongs to a class of thienopyridine molecules which targets the $\mathrm{P}_{2} \mathrm{Y}_{12}$ receptor on platelets and it is commonly used in combination with the COX-1 inhibitor acetylsalicylic acid (ASA). However, the effect of these treatments is variable amongst patients, highlighting a need for a refinement of this class of $\mathrm{P}^{2} \mathrm{Y}_{12}$ inhibitor. The aim of this study was to assess the efficacy of six novel thienopyridine derivatives synthesised by our group by examining their potential as in-vitro inhibitors of platelet function.

Methods Healthy human platelets were isolated and incubated with novel thienopyridine compounds (DJ0081, DJ0199, DJ0021, DJ0206, DJ0171, DJ0097) $(10 \mu \mathrm{M}, 30 \mathrm{~min})$ prior to stimulation with ADP $(10 \mu \mathrm{M})$ and analysis of alpha granule secretion (CD62P expression), GPIIbIIIa activation (PAC1 expression) and platelet leukocyte aggregate (PLA) formation using flow cytometry. Furthermore, light transmission aggregometry (LTA) was used to assess ADP-stimulated aggregation after these treatments. Synergy with ASA $(30 \mu \mathrm{M})$ was also analysed by LTA following incubation with ASA and thienopyridine. All results were compared to ADP-stimulated samples and samples treated with clopidogrel $(10 \mu \mathrm{M}, 30 \mathrm{~min})$ prior to ADP stimulation.

Results All six novel compounds demonstrated a significant reduction in ADP-mediated platelet aggregation $(\mathrm{p}<0.001)$, CD62P expression $(\mathrm{p}<0.001)$, PAC1 expression $(\mathrm{p}<0.01)$ and PLA formation $(p<0.05)$. These compounds were also shown to enhance the inhibitory effects of ASA. DJ0171 and DJ0199 were particularly potent, displaying greater inhibitory effect than clopidogrel.

Conclusion The study demonstrates the potential for new thienopyridine compounds as modulators of platelet function and points to the possibility of future use in patients at risk of platelet hyperactivity and thrombosis.

\section{DEVELOPMENT OF A NOVEL SOFTWARE PACKAGE FOR HIGH-THROUGHPUT PROCESSING AND ANALYSIS OF CARDIAC OPTICAL MAPPING DATA}

Christopher O'Shea ${ }^{1,2,3},{ }^{2}$ Andrew Holmes, ${ }^{2}$ Ting Yue Yu, ${ }^{4}$ James Winter, ${ }^{5}$ Joao Correia, ${ }^{2}$ Paulus Kirchhof, ${ }^{2}$ Larissa Fabritz, ${ }^{3}$ Kashif Rajpoot, ${ }^{2}$ Davor Pavlovic. ${ }^{1}$ EPSRC Centre for Doctoral Training in Physical Sciences for Health, University of Birmingham, Birmingham,UK; ${ }^{2}$ Institute of Cardiovascular sciences, University of Birmingham, Birmingham, UK: ${ }^{3}$ School of Computer science, University of Birmingham, Birmingham, UK; ${ }^{4}$ Cardiovascular Division, The Rayne Institute, King's College London, UK; ${ }^{5}$ Cairn Research, Graveney Road, Faversham, UK

\subsection{6/heartjnl-2017-311726.186}

Background Optical mapping is a powerful research tool that is revolutionising study of cardiac electrophysiology. However, processing and analysis of optical mapping data is computationally challenging to design and implement, a difficulty further enhanced by novel camera technology providing higher temporal and spatial resolution. We have previously developed algorithms capable of objective processing and analysis of electrophysiological parameters acquired using a second-generation complementary metal-oxide semiconductor camera. ${ }^{1}$ Here we report development of improved algorithms packaged in a user-friendly graphical interface, capable of high-throughput processing and analysis of voltage and calcium optical mapping data from a wide spectrum of cameras. Functionality and processing speed is further improved through automated recognition of pacing frequency and analysis of activation and repolarisation of electrophysiological parameters at high spatiotemporal resolution $(200 \times 2400$ pixels; sampling rate $1 \mathrm{kHz}$ ). Processing options allow averaging of multiple beats as well as individual beat segmentation within the whole experimental trace, thus allowing for study of dynamic changes in key parameters such as action potential duration (APD), activation times, conduction velocity (CV) and phase mapping.

Methods and results We have compared how our software performs in terms of analysis and processing time with our 


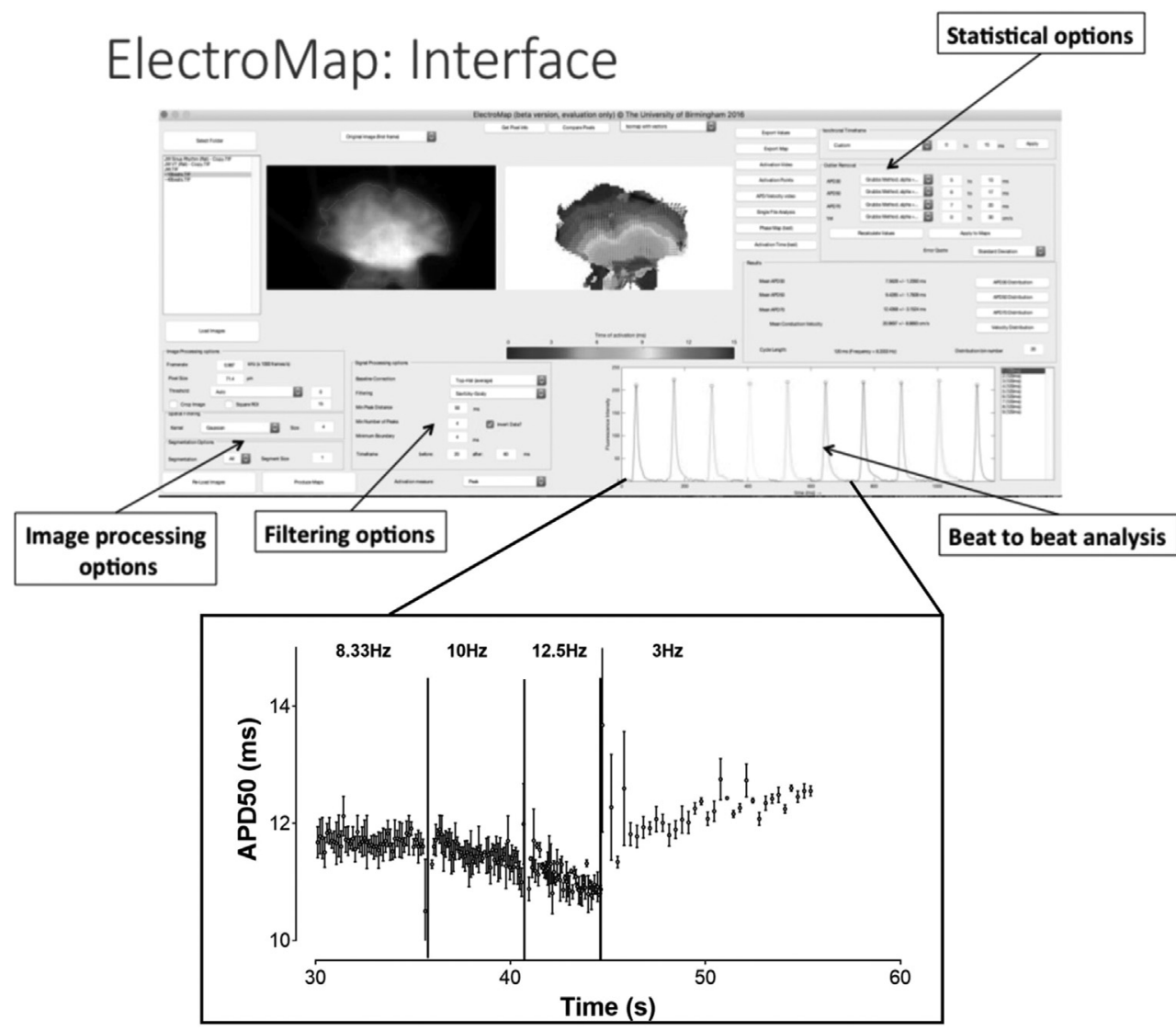

Abstract 188 Figure 1 Graphical user interface offering parameter options for image processing, filtering and statistics. Software allows signal segmentation into single or multiple beats for analysis. Inset: Beat-to-beat analysis of frequency-dependent changes in action potential duration (APD50) in paced isolated murine atria.

previously published methods. ${ }^{1}$ Results remain consistent between both methods (APD50 $=10.41 \pm 0.62$ vs 10.36 $\pm 0.63 \mathrm{~ms} ; \mathrm{CV}=24.3 \pm 1.68$ vs $23.3 \pm 1.69 \mathrm{~cm} / \mathrm{s}$, new software vs published methods; $\mathrm{n}=5$, murine atria). Substantial improvements in processing speed (up to 4 times) are achieved, compared to our previously published methods. This improvement, coupled with automatic recognition and segmentation of whole experiments by cycle length, enables analysis of frequency dependent beat-to-beat changes in APD, CV and activation times. We demonstrate dynamic beat-to-beat frequency-dependent changes in APD50 in isolated superfused murine atria over a long experimental timeframe. As expected, increased frequency reduces APD50 (3 Hz=12.56 \pm 0.08 ; $8.33 \mathrm{~Hz}=11.78 \pm 0.29 ; \quad 10 \mathrm{~Hz}=11.47 \pm 0.3 ; \quad 12.5 \mathrm{~Hz}=10.88$ $\pm 0.29 \mathrm{~ms} ; \mathrm{n}=3$ ), however, extensive beat-to-beat analysis allows investigation of electophysiological changes over whole experimental protocols, potentially providing crucial insight into novel arrhythmic mechanisms.

Conclusions We have developed high-throughput software for analysis and processing of optical mapping imaging data compatible with a wide range of optical mapping cameras/systems. Our software offers enhanced processing speeds of key electrophysiological parameters across the heart and allows beatto-beat analysis of large computationally challenging datasets.

\section{REFERENCE}

1. Yue et al. Prog Biophys Mol Biol. 2014 Aug;115(2-3):340-8.

\section{ALTERED PLATELET FUNCTION AND THROMBOSIS IN FETAL GROWTH RESTRICTION}

${ }^{1}$ Olube Obe, ${ }^{2}$ Helen Bischof, ${ }^{1}$ Nina Dempsey-Hibbert, ${ }^{2}$ Paul Brownbill, 'Sarah Jones*. ${ }^{1}$ Manchester Metropolitan University; ${ }^{2}$ University of Manchester

\subsection{6/heartjnl-2017-311726.187}

Background Fetal growth restriction (FGR) affects 3\%-8\% of pregnancies and is associated with increased risk of stillbirth and perinatal morbidity, in addition to increased risk of cardiovascular disease later in life. In severe cases of FGR, vascular resistance in the placenta is increased and blood flow reduced, compromising oxygen and nutrient exchange between mother and fetus. Hypoxia and endothelial dysfunction, have both been demonstrated in FGR, and both have the ability to alter thrombosis and haemostasis. The aim of this study was to determine whether there is increased platelet activation and thrombosis in FGR which may contribute to its pathogenesis. Methods Dual ex-vivo perfusions were performed on placentas from healthy pregnancies $(n=10)$ and those affected by FGR $(n=11)$. Following perfusion $(\sim 5$ hour $)$, placental cross-sections were processed and stained with $\mathrm{H}$ and $\mathrm{E}$ and Martius Scarlet Blue for blind-randomised histological analysis or immunofluorescence to analyse levels of fibrin and tissue factor. Fetal blood samples were collected from the umbilical vein immediately following delivery ( $n=10$ healthy; $n=8$ FGR) and platelet function analysed using the PFA200

Results Placental sections from pregnancies affected by FGR exhibited significantly more thrombi $(p<0.01)$ within the fetal 